TRANSACTIONS OF THE

AMERICAN MATHEMATICAL SOCIETY

Volume 352, Number 9 , Pages 3937-3956

S 0002-9947(00)02532-0

Article electronically published on May 22, 2000

\title{
LOCAL BOUNDARY RIGIDITY OF A COMPACT RIEMANNIAN MANIFOLD WITH CURVATURE BOUNDED ABOVE
}

\author{
CHRISTOPHER B. CROKE, NURLAN S. DAIRBEKOV, \\ AND VLADIMIR A. SHARAFUTDINOV
}

\begin{abstract}
This paper considers the boundary rigidity problem for a compact convex Riemannian manifold $(M, g)$ with boundary $\partial M$ whose curvature satisfies a general upper bound condition. This includes all nonpositively curved manifolds and all sufficiently small convex domains on any given Riemannian manifold. It is shown that in the space of metrics $g^{\prime}$ on $M$ there is a $C^{3, \alpha_{-}}$ neighborhood of $g$ such that $g$ is the unique metric with the given boundary distance-function (i.e. the function that assigns to any pair of boundary points their distance - as measured in $M$ ). More precisely, given any metric $g^{\prime}$ in this neighborhood with the same boundary distance function there is diffeomorphism $\varphi$ which is the identity on $\partial M$ such that $g^{\prime}=\varphi^{*} g$. There is also a sharp volume comparison result for metrics in this neighborhood in terms of the boundary distance-function.
\end{abstract}

\section{Statement of the Result}

The general boundary rigidity problem reads: to which extent is a Riemannian metric on a compact manifold with boundary determined from the distances between boundary points? More precisely, it can be formulated as follows.

Let $(M, g)$ be a compact Riemannian manifold with boundary $\partial M$. Let $g^{\prime}$ be another Riemannian metric on $M$. We say that $g$ and $g^{\prime}$ have the same boundary distance-function if $d_{g}(x, y)=d_{g^{\prime}}(x, y)$ for arbitrary boundary points $x, y \in \partial M$, where $d_{g}$ (resp. $d_{g^{\prime}}$ ) represents distance in $M$ with respect to $g$ (resp. $\left.g^{\prime}\right)$. It is easy to give examples of pairs of metrics with the same boundary distance-function. Indeed, if $\varphi: M \rightarrow M$ is an arbitrary diffeomorphism of $M$ onto itself which is the identity on the boundary, then the metrics $g$ and $g^{\prime}=\varphi^{*} g$ have the same boundary distance-function. Here $g^{\prime}=\varphi^{*} g$ is the pull-back of $g$ under $\varphi$ (i.e., for arbitrary vectors $\xi, \eta \in T_{x} M$ we have $\langle\xi, \eta\rangle_{x}^{\prime}=\left\langle\varphi_{*} \xi, \varphi_{*} \eta\right\rangle_{\varphi(x)}$, where $\varphi_{*}: T_{x} M \rightarrow T_{\varphi(x)} M$ is the differential of $\varphi$ at $x$ and $\langle$,$\rangle (resp. \langle,\rangle^{\prime}$ ) is the inner product with respect to the metric $g\left(\right.$ resp. $\left.\left.g^{\prime}\right)\right)$.

We say that a compact Riemannian manifold is boundary rigid if this is the only type of nonuniqueness. More precisely, $(M, g)$ is boundary rigid if, for every

Received by the editors August 10, 1998.

2000 Mathematics Subject Classification. Primary 53C65, 53C22, 53C20; Secondary 58C25, $58 \mathrm{C} 40$.

The first and third authors were supported by CRDF, Grant RM2-143.

The first author was also supported by NSF grants \#MDS-9505175 and \#MDS-9626232.

The third author was also supported by INTAS - RFBR, Grant 95-0763. 
Riemannian metric $g^{\prime}$ on $M$ with the same boundary distance-function as $g$, there is a diffeomorphism $\varphi: M \rightarrow M$ which is the identity on the boundary and for which $g^{\prime}=\varphi^{*} g$. It was conjectured in Cr1 that, $S G M$-manifolds ( $S G M$ - "strong geodesic minimizing") are always boundary rigid. This conjecture holds in a number of cases (see for example [Cr2], [Ml], and [Ot]).

In the present article we prove "local" boundary rigidity for a compact dissipative Riemannian manifold $(M, g)$ with a certain upper bound of the curvature. The word "local" means that we presume $g^{\prime}$ to be sufficiently close to $g$. A similar result was established recently in [SU] for $M$ a convex domain in $\mathbb{R}^{n}$ and the metrics $g$ and $g^{\prime}$ sufficiently close to the Euclidean metric.

We recall that $(M, g)$ is a compact dissipative Riemannian manifold (CDRM) if $M$ is compact, the boundary $\partial M$ is strictly convex, and for every point $x \in M$ and every nonzero vector $\xi \in T_{x} M$ the maximal geodesic $\gamma_{x, \xi}(t)$, satisfying the initial conditions $\gamma_{x, \xi}(0)=x$ and $\dot{\gamma}_{x, \xi}(0)=\xi$, is defined on some bounded interval $\left[\tau_{-}(x, \xi), \tau_{+}(x, \xi)\right]$.

Let $\Omega M=\left\{(x, \xi)\left|x \in M, \xi \in T_{x} M,\right| \xi \mid=1\right\}$ stand for the unit sphere bundle of $M$. The boundary $\partial \Omega M$ is the union of two submanifolds

$$
\partial_{ \pm} \Omega M=\{(x, \xi) \in \Omega M \mid x \in \partial M, \pm\langle\xi, \nu(x)\rangle \geq 0\}
$$

of inward and outward vectors. Here $\nu$ is the unit outward normal to $\partial M$.

Given $(x, \xi) \in \Omega M$, we denote by $K(x, \xi)$ the maximum of the sectional curvatures of all two-planes $\sigma \subset T_{x} M$ such that $\xi \in \sigma$.

For $(M, g)$ a CDRM, we define the following invariant:

$$
k^{+}(M, g)=\sup _{(x, \xi) \in \partial_{-} \Omega M} \int_{0}^{\tau_{+}(x, \xi)} t K^{+}\left(\gamma_{x, \xi}(t), \dot{\gamma}_{x, \xi}(t)\right) d t,
$$

where

$$
K^{+}(x, \xi)=\max \{0, K(x, \xi)\} .
$$

In particular, $k^{+}(M, g)=0$ if $(M, g)$ is nonpositively curved.

Given a natural number $k$ and a real number $\alpha, 0<\alpha<0$, we denote by $\operatorname{Diff}_{0}^{k, \alpha}(M)$ the set of all diffeomorphisms of $M$ onto itself that are the identity on the boundary and are given by functions of class $C_{l o c}^{k, \alpha}$ in local coordinates of $M$. We endow $\operatorname{Diff}_{0}^{k, \alpha}(M)$ with the $C^{k, \alpha}$-topology, defining some $C^{k, \alpha}$-norm by means of a finite atlas and a subordinate partition of unity. The resultant topology is clearly independent of the choice of the norm.

We let $C^{k, \alpha}\left(S^{2} \tau_{M}^{\prime}\right)$ stand for the space of $C^{k, \alpha}$-smooth covariant symmetric tensor fields of degree 2 on $M$. We endow $C^{k, \alpha}\left(S^{2} \tau_{M}^{\prime}\right)$ with the natural $C^{k, \alpha_{-}}$ topology. Then $C^{k, \alpha}\left(S^{2} \tau_{M}^{\prime}\right)$ becomes a topological Banach space, i.e., a topological vector space whose topology can be defined by some norm making it a Banach space.

Now, we are in a position to formulate our main result.

Theorem 1.1. Let a $C D R M(M, g)$ satisfy the condition

$$
k^{+}(M, g)<1 / 3 \text {. }
$$

Then there is a neighborhood $W \subset C^{3, \alpha}\left(S^{2} \tau_{M}^{\prime}\right)$ of $g$, with any $0<\alpha<1$, such that if a metric $g^{\prime} \in W$ has the same boundary distance-function as $g$, then there exists a diffeomorphism $\varphi: M \rightarrow M$ in $\operatorname{Diff}_{0}^{3, \alpha}(M)$ such that $g^{\prime}=\varphi^{*} g$; moreover, $\varphi$ tends to the identity as $g^{\prime}$ tends to $g$ (both in $C^{3, \alpha}$-topology). 
Remark. Condition (1.2) implies the simplicity of $(M, g)$; cf. p. 120 of [Sh2]. This means that every pair of points $p, q$ in $M$ can be joined by a unique minimizing geodesic segment $\gamma_{p q}$ that varies smoothly with $p$ and $q$. Inequality (1.2) holds for instance when $M$ is nonpositively curved or is a sufficiently small convex piece of an arbitrary Riemannian manifold.

The "infinitesimal" version of this "local" theorem was proved in [Sh1]. The infinitesimal version says in particular that if $g$ satisfies the condition of the theorem and if $g_{t}$ is a smooth one parameter family of Riemannian metrics on $M$ with $g_{0}=g$, all having the same boundary distance-function, then $g_{t}$ is isometric to $g$. "Local" uniqueness theorems can be considered part of a program for proving finiteness theorems (the other part being compactness results).

The problem considered here is closely related to the isospectral problem (length and eigenvalue spectra) for compact manifolds of negative curvature without boundary. In [Cr-Sh following the earlier work of GK] the "infinitesimal" version was proved. Also see [Sh3] for results in the Anosov case. However, not all of the techniques used here can be extended to that case (for which a number of compactness results already exists; see for example $\underline{\mathrm{An}}$ and $[\mathrm{BPP}]$ ). However the results of Section 2 do extend. The main problem in extending these results to the no boundary case lies in the non-existence of an appropriate "approximate Livčic theorem".

In the next few paragraphs we explain how the paper is organized. Each section treats a different aspect of the problem and many sections work more generally than when $d_{g}=d_{g^{\prime}}$. For example, Sections 2 and 4 deal with any two sufficiently close metrics while Section 3 deals with a metric $g^{\prime}$ near a simple metric $g$ such that $d_{g^{\prime}}(x, y) \geq d_{g}(x, y)$ for all $x, y$ on the boundary.

In Section 2 we "shift" any tensor $g^{\prime}$ which is sufficiently close to a given metric $g$ to a solenoidal one with respect to $g$. That is, we find a diffeomorphism $\varphi \in$ $\operatorname{Diff}_{0}^{3, \alpha}(M)$ such that the pull-back $g^{1}=\varphi^{*} g^{\prime}$ of $g^{\prime}$ is a solenoidal tensor field with respect to $g$ (i.e., the $g$-divergence of $g^{1}$ is 0 ).

In Section [3, we show that if $g^{1}$ is sufficiently close to a simple metric $g$ and if, for all pairs $x$ and $y$ on the boundary, $d_{g^{1}}(x, y) \geq d_{g}(x, y)$, then the ray transform If of the tensor $f=g^{1}-g$ is nonnegative (i.e. If $(\gamma) \geq 0$ for every geodesic ray $\gamma$ from a boundary point to a boundary point). Also using Santaló's formula we see that $\lambda \equiv(g, f)_{L_{2}\left(S^{2} \tau_{M}^{\prime}\right)} \geq 0$.

In Section 4, we consider the volume of metrics $g^{1}$ which are sufficiently close to a given metric $g$. We show that if $\operatorname{Vol}\left(g^{1}\right) \leq \operatorname{Vol}(g)$, then the tensor $f=g^{1}-g$ satisfies $\lambda \leq \frac{2}{3}\|f\|_{L_{2}\left(S^{2} \tau_{M}^{\prime}\right)}^{2}$.

In Section 5, we consider metrics $g^{1}$ close to a given dissipative metric $g$ which induce the same Riemannian metric on the boundary as the one induced by $g$. The ray transform of $f=g^{1}-g$ satisfies a number of useful properties that are exploited in Section 6.

In Section 6 , we complete the proof of the main theorem with the help of Pestov's identity. In fact we show

Proposition 1.2. For any metric $g$ satisfying the assumptions of Theorem 1.1 there is a neighborhood $W \subset C^{3, \alpha}\left(S^{2} \tau_{M}^{\prime}\right)$ of $g$, with any $0<\alpha<1$, such that if a metric $g^{\prime} \in W$ induces the same Riemannian metric on the boundary as $g$ and $d_{g^{\prime}}(x, y) \geq d_{g}(x, y)$ for all boundary points $x$ and $y$, then $\operatorname{Vol}\left(g^{\prime}\right) \geq \operatorname{Vol}(g)$ with equality if and only if $g^{\prime}$ is isometric to $g$. 
The main theorem follows directly from this since if $g^{\prime}$ and $g$ have the same boundary distance-function, then they induce the same Riemannian metric on the boundary and they have the same volume.

Proposition 1.2 may be of some independent interest since little is understood about how inequalities between the boundary distance functions might relate the volumes of Riemannian manifolds with boundary (see for example Gromov's notion of the filling volume $[\mathrm{Gr}]$ ). The corresponding statement for the compact without boundary case would be: If $g$ is a metric on a compact negatively curved manifold $M$ and $g^{\prime}$ is a metric sufficiently close to $g$ and such that the $g^{\prime}$-length of each free homotopy class is $\geq$ the $g$-length, then $\operatorname{Vol}\left(g^{\prime}\right) \geq \operatorname{Vol}(g)$ with equality holding if and only if $g^{\prime}$ is isometric to $g$. This statement remains an open question, but the results in this paper lend it support.

\section{Shift of A tensor Field to A SOlenoidal ONE}

Theorem 2.1. Let $(M, g)$ be a compact Riemannian manifold with convex boundary and let $k \geq 2$ be an integer and $0<\alpha<1$ a real number. Then for every neighborhood $U \subset \operatorname{Diff}_{0}^{k, \alpha}(M)$ of the identity there is a neighborhood $W \subset C^{k, \alpha}\left(S^{2} \tau_{M}^{\prime}\right)$ of the metric tensor $g$ such that for every metric $g^{\prime} \in W$ there exists a diffeomorphism $\varphi \in U$ for which the tensor field $\varphi^{*} g^{\prime}$ is solenoidal; i.e., $\delta\left(\varphi^{*} g^{\prime}\right)=0$, where $\delta$ is the divergence in the metric $g$.

Remark. The assumption that the boundary is convex slightly simplifies the proof of the theorem but is not essential for its validity. A similar theorem holds for a closed $(M, g)$ under the assumption that there exists a dense geodesic in $\Omega M$.

The proof consists in applying a Banach space version of the implicit function theorem. To this end, we first of all must realize some neighborhood of the identity in $\operatorname{Diff}_{0}^{k, \alpha}(M)$ as an open set in a Banach space.

Denote by $C_{0}^{k, \alpha}\left(\tau_{M}\right)$ the topological Banach space of vector fields of class $C^{k, \alpha}$ on $M$ which vanish on $\partial M$. Let $\Omega$ be the open neighborhood of the zero in $C_{0}^{k, \alpha}\left(\tau_{M}\right)(k \geq 1)$ which consists of the vector fields $v$ satisfying the inequality $|\nabla v|<1$. This inequality and the boundary condition $\left.v\right|_{\partial M}=0$ imply that $|v(x)| \leq \operatorname{dist}(x, \partial M)$ for all $x \in M$. Therefore, the mapping

$$
e_{v}: M \rightarrow M, \quad e_{v}(x)=\exp _{x} v(x)
$$

is well-defined for all $v \in \Omega$. It is easy to check that there is some smaller neighborhood $\Omega^{\prime} \subset \Omega$ of zero in $C_{0}^{k, \alpha}\left(\tau_{M}\right)$ such that $e_{v} \in \operatorname{Diff}_{0}^{k, \alpha}(M)$ for $v \in \Omega^{\prime}$. The mapping

$$
\Omega^{\prime} \rightarrow \operatorname{Diff}_{0}^{k, \alpha}(M), \quad v \mapsto e_{v}
$$

is continuous. The inverse of $(2.2), \varphi \mapsto v_{\varphi}$, is defined for $\varphi \in \operatorname{Diff}_{0}^{k, \alpha}(M)$ sufficiently close to the identity as follows: $v_{\varphi}(x)=\dot{\gamma}(0)$, where $\gamma:[0,1] \rightarrow M$ is the geodesic such that $\gamma(0)=x$ and $\gamma(1)=\varphi(x)$; the existence of this geodesic is guaranteed by the convexity of the boundary. We thus establish that $(2.2)$ is a homeomorphism of the neighborhood $\Omega^{\prime}$ of the zero in the Banach space $C_{0}^{k, \alpha}\left(\tau_{M}\right)$ onto some neighborhood of the identity in the space Diff ${ }_{0}^{k, \alpha}(M)$. Therefore, the theorem will be proven once we prove the following assertion.

Lemma 2.2. Under the conditions of Theorem 2.1, let $\Omega \subset C_{0}^{k, \alpha}\left(\tau_{M}\right)$ be a neighborhood of zero such that the mapping (2.1) is defined for all $v \in \Omega$. Then there 
exists a neighborhood $G \subset C_{0}^{k, \alpha}\left(S^{2} \tau_{M}^{\prime}\right)$ of zero and a continuous mapping $\beta: G \rightarrow \Omega$ such that $\beta(0)=0$ and the tensor field $\left(e_{\beta(f)}\right)^{*}(g+f)$ is solenoidal (in the metric g) for all $f \in G$.

Proof. Consider the mapping

$$
F: \Omega \times C^{k, \alpha}\left(S^{2} \tau_{M}^{\prime}\right) \rightarrow C^{k-2, \alpha}\left(\tau_{M}^{\prime}\right)
$$

defined by

$$
F(v, f)=\delta\left(e_{v}^{*}(g+f)\right), \quad v \in \Omega \subset C_{0}^{k, \alpha}\left(\tau_{M}\right), f \in C^{k, \alpha}\left(S^{2} \tau_{M}^{\prime}\right) .
$$

We need to show that $F$ is continuous and has continuous partial derivatives $F_{v}^{\prime}$ and $F_{f}^{\prime}$. To this end, represent $F$ as the composition

$$
F(v, f)=\delta R(v, g+f),
$$

where $\delta: C^{k-1, \alpha}\left(S^{2} \tau_{M}^{\prime}\right) \rightarrow C^{k-2, \alpha}\left(\tau_{M}^{\prime}\right)$ is the divergence in the metric $g$ and the mapping

$$
R: \Omega \times C^{k, \alpha}\left(S^{2} \tau_{M}^{\prime}\right) \rightarrow C^{k-1, \alpha}\left(S^{2} \tau_{M}^{\prime}\right)
$$

is defined by

$$
R(v, f)=e_{v}^{*} f .
$$

Since $\delta$ is a first order linear differential operator, we have

$$
F_{v}^{\prime}(v, f)=\delta R_{v}^{\prime}(v, g+f), \quad F_{f}^{\prime}(v, f)=\delta R_{f}^{\prime}(v, g+f) .
$$

Hence, the matter is reduced to verifying the continuity of the function $R$ and of its derivatives $R_{v}^{\prime}$ and $R_{f}^{\prime}$.

Let $\left(x^{1}, \ldots, x^{n}\right)$ be a local coordinate system on $M$ with domain $U \subset M$. For $x \in U$ and a sufficiently small vector $\xi \in T_{x} M$, the point $\exp _{x} \xi$ belongs to $U$ as well; we denote the coordinates of this point by $\left(E^{1}(x, \xi), \ldots, E^{n}(x, \xi)\right)$. According to $(2.1)$, the point $e_{v}(x)$ has coordinates $\left(e_{v}^{1}(x), \ldots, e_{v}^{n}(x)\right)$ with

$$
e_{v}^{i}(x)=E^{i}(x, v(x)) \text {. }
$$

Now, (2.7) is rewritten in coordinates as

$$
(R(v, f))_{i j}=\frac{\partial e_{v}^{p}}{\partial x^{i}} \frac{\partial e_{v}^{q}}{\partial x^{j}} f_{p q} \circ e_{v} .
$$

For every vector field $v \in C_{0}^{k, \alpha}\left(\tau_{M}\right)$, the function $e_{v}^{p}(x)$ is of class $C^{k, \alpha}$. The fact that the right-hand side of (2.10) lies in the space $C^{k-1, \alpha}\left(S^{2} \tau_{M}^{\prime}\right)$ and that it has continuous dependence on $(v, f)$ follow from the two facts:

(a) if $\varphi, \psi \in C^{k, \alpha}$, then the product $\varphi \psi$ also belongs to $C^{k, \alpha}$ and the mapping $C^{k, \alpha} \times C^{k, \alpha} \rightarrow C^{k, \alpha},(\varphi, \psi) \mapsto \varphi \psi$, is continuous;

(b) if $\varphi, \psi \in C^{k, \alpha}(k \geq 1)$ and the composition $\varphi \circ \psi$ is defined, then $\varphi \circ \psi \in C^{k, \alpha}$ and the mapping $C^{k, \alpha} \times C^{k, \alpha} \rightarrow C^{k, \alpha},(\varphi, \psi) \mapsto \varphi \circ \psi$, is continuous.

Since the mapping (2.6) is linear in $f$, the partial derivative $R_{f}^{\prime}$ is given by the expression $R_{f}^{\prime}(v, f) \widetilde{f}=e_{v}^{*} \widetilde{f}$ and its continuity ensues from the same arguments as for $R$. 
Differentiating (2.10) with respect to $v$, we find (using $f_{p q}=f_{q p}$ ) the partial derivative $R_{v}^{\prime}$ :

$$
\begin{array}{r}
\left(R_{v}^{\prime}(v, f) \widetilde{v}\right)_{i j}=\left(\frac{\partial e_{v}^{p}}{\partial x^{i}} \frac{\partial\left(D e_{v}^{q}\right)}{\partial x^{j}}+\frac{\partial e_{v}^{p}}{\partial x^{j}} \frac{\partial\left(D e_{v}^{q}\right)}{\partial x^{i}}\right) f_{p q} \circ e_{v} \\
+\left(D e_{v}^{r}\right) \frac{\partial e_{v}^{p}}{\partial x^{i}} \frac{\partial e_{v}^{q}}{\partial x^{j}} \frac{\partial f_{p q}}{\partial x^{r}} \circ e_{v}
\end{array}
$$

where $D e_{v}^{i}$ is the variation of the function $e_{v}^{i}$ which by (2.9) is given by the expression

$$
D e_{v}^{i}=\frac{\partial E^{i}}{\partial \xi^{r}}(x, v(x)) \widetilde{v}^{r}(x) .
$$

On using (2.11) and (2.12), the continuity of $R_{v}^{\prime}$ follows from the same arguments as above.

We now compute $F_{v}^{\prime}(0,0)$. Setting $v=0, f=g$ in (2.11), (2.12) and using the relations

$$
\left.e_{v}^{i}\right|_{v=0}=x^{i}, \quad \frac{\partial E^{i}}{\partial \xi^{r}}(x, 0)=\delta_{r}^{i}
$$

we find

$$
\left(R_{v}^{\prime}(0, g) \widetilde{v}\right)_{i j}=g_{i p} \frac{\partial \widetilde{v}^{p}}{\partial x^{j}}+g_{j p} \frac{\partial \widetilde{v}^{p}}{\partial x^{i}}+\frac{\partial g_{i j}}{\partial x^{p}} \widetilde{v}^{p} .
$$

Rewriting the partial derivatives $\partial \widetilde{v}^{p} / \partial x^{i}$ in terms of the covariant derivatives $\nabla_{i} \widetilde{v}^{p}=\partial \widetilde{v}^{p} / \partial x^{i}+\Gamma_{i q}^{p} \widetilde{v}^{q}$, we arrive at the equality

$$
\left(R_{v}^{\prime}(0, g) \widetilde{v}\right)_{i j}=\nabla_{i} \widetilde{v}_{j}+\nabla_{j} \widetilde{v}_{i}=2(d \widetilde{v})_{i j}
$$

where $\widetilde{v}_{i}=g_{i j} \widetilde{v}^{j}$ and $d=\sigma \nabla$ is the symmetric part of the covariant derivative in the metric $g$. Thus we have shown that

$$
R_{v}^{\prime}(0, g)=2 d
$$

From (2.8) and (2.13) we see that

$$
F_{v}^{\prime}(0,0)=\delta R_{v}^{\prime}(0, g)=2 \delta d
$$

As shown in Section 3.3 of [Sh2], the Dirichlet problem for the operator $\delta d$ is elliptic and has zero kernel and cokernel in appropriate Sobolev spaces. Now, the Schauder-type estimates of [ADN] for elliptic boundary value problems in the spaces $C^{k, \alpha}$ imply that the operator

$$
F_{v}^{\prime}(0,0)=\delta d: C_{0}^{k, \alpha}\left(\tau_{M}\right) \rightarrow C^{k-2, \alpha}\left(S^{2} \tau_{M}^{\prime}\right)
$$

has a continuous inverse.

We have thus verified that the function (2.3) satisfies all conditions of the implicit function theorem $[\mathrm{KA}$. This theorem guarantees local solvability of the equation $F(v, f)=0$ in $v$ over a neighborhood of the point $(v, f)=(0,0)$, which completes the proof of Theorem 2.1. 


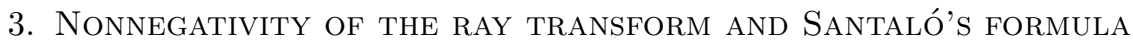

In what follows, some notions (the ray transform, the vertical and horizontal derivatives, the Pestov identity, and so on) are used without definitions. The definitions can be found in Sh2.

For a tensor field $f \in C^{2}\left(S^{2} \tau_{M}^{\prime}\right)$ on a simple Riemannian manifold we define the ray transform If as a map from $\{\gamma \mid \gamma:[0,1] \rightarrow M$ is a geodesic segment between boundary points $\}$ to the reals defined by

$$
I f(\gamma)=\int_{0}^{1} f_{i j}(\gamma(t)) \dot{\gamma}^{i}(t) \dot{\gamma}^{j}(t) d t .
$$

The first goal of this section is to show:

Proposition 3.1. If $(M, g)$ is a simple Riemannian manifold, then there exists $\varepsilon>0$ such that if $f \in C^{2}\left(S^{2} \tau_{M}^{\prime}\right)$ satisfies

$$
\|f\|_{C^{2}\left(S^{2} \tau_{M}^{\prime}\right)}<\varepsilon
$$

and if for every pair $x, y \in \partial M$ the metric $g^{1}=g+f$ satisfies

$$
d_{g^{1}}(x, y) \geq d_{g}(x, y)
$$

then If $\geq 0$.

Let $(M, g)$ be a simple Riemannian manifold (which implies in particular that the boundary of $M$ is strictly convex). Let $\varepsilon>0$ be so small that for every $f \in C^{2}\left(S^{2} \tau_{M}^{\prime}\right)$ such that

$$
\|f\|_{C^{2}\left(S^{2} \tau_{M}^{\prime}\right)}<\varepsilon
$$

the metric

$$
g^{\tau}=g+\tau f \quad(0 \leq \tau \leq 1)
$$

is also simple for every $\tau \in[0,1]$.

Fix two points $p, q \in \partial M$ and let

$$
\gamma_{\tau}:[0,1] \rightarrow M, \quad \gamma_{\tau}(0)=p, \quad \gamma_{\tau}(1)=q
$$

be the geodesic of $g^{\tau}$ between $p$ and $q$. The simplicity of the metrics $g^{\tau}$ guarantees that the $\gamma_{\tau}$ vary differentiably. Denote the energy of $\gamma_{\tau}$ by $E(\tau)$ :

$$
E(\tau)=\int_{\gamma_{\tau}} g^{\tau} d t=\int_{0}^{1} g_{i j}^{\tau}\left(\gamma_{\tau}(t)\right) \dot{\gamma}_{\tau}^{i}(t) \dot{\gamma}_{\tau}^{j}(t) d t .
$$

Then $E(\tau)$ is a $C^{2}$-smooth function on $[0,1]$ and (since $\left.\frac{d}{d \beta}\right|_{\beta=\tau} \int_{\gamma_{\beta}} g^{\tau} d t=0$ )

$$
E^{\prime}(\tau)=\left(I^{\tau} f\right)\left(\gamma_{\tau}\right)=\int_{0}^{1} f_{i j}\left(\gamma_{\tau}(t)\right) \dot{\gamma}_{\tau}^{i}(t) \dot{\gamma}_{\tau}^{j}(t) d t
$$

where $I^{\tau}$ is the ray transform in the metric $g^{\tau}$.

Proposition 3.1 now follows from:

Lemma 3.2. The function $E(\tau)$ is concave on $[0,1]$; i.e., $E^{\prime \prime}(\tau) \leq 0$. 
Proof. Let $0 \leq \tau<\tau^{\prime} \leq 1$. Since $\gamma_{\tau}$ is an extremal for $g^{\tau}$, we can write

$$
\begin{aligned}
E\left(\tau^{\prime}\right)= & \int_{\gamma_{\tau^{\prime}}} g^{\tau^{\prime}} d t=\int_{\gamma_{\tau^{\prime}}}\left(g^{\tau}+\left(\tau^{\prime}-\tau\right) f\right) d t=\int_{\gamma_{\tau^{\prime}}} g^{\tau} d t+\left(\tau^{\prime}-\tau\right) \int_{\gamma_{\tau^{\prime}}} f d t \\
& \geq \int_{\gamma_{\tau}} g^{\tau} d t+\left(\tau^{\prime}-\tau\right) \int_{\gamma_{\tau^{\prime}}} f d t=E(\tau)+\left(\tau^{\prime}-\tau\right) E^{\prime}\left(\tau^{\prime}\right) .
\end{aligned}
$$

Thus,

$$
E^{\prime}\left(\tau^{\prime}\right) \leq \frac{E\left(\tau^{\prime}\right)-E(\tau)}{\tau^{\prime}-\tau}
$$

Similarly

$$
\begin{gathered}
E(\tau)=\int_{\gamma_{\tau}} g^{\tau} d t=\int_{\gamma_{\tau}}\left(g^{\tau^{\prime}}-\left(\tau^{\prime}-\tau\right) f\right) d t=\int_{\gamma_{\tau}} g^{\tau^{\prime}} d t-\left(\tau^{\prime}-\tau\right) \int_{\gamma_{\tau}} f d t \\
\quad \geq \int_{\gamma_{\tau^{\prime}}} g^{\tau^{\prime}} d t-\left(\tau^{\prime}-\tau\right) \int_{\gamma_{\tau}} f d t=E\left(\tau^{\prime}\right)-\left(\tau^{\prime}-\tau\right) E^{\prime}(\tau) .
\end{gathered}
$$

Thus,

$$
E^{\prime}(\tau) \geq \frac{E\left(\tau^{\prime}\right)-E(\tau)}{\tau^{\prime}-\tau} .
$$

Comparing (3.4) and (3.5), we obtain $E^{\prime}(\tau) \geq E^{\prime}\left(\tau^{\prime}\right)$, completing the proof of the lemma.

We now recall the Santaló formula [S1]:

$$
\int_{\Omega M} F(x, \xi) d \Sigma^{2 n-1}(x, \xi)=\int_{\partial_{+} \Omega M}\langle\xi, \nu(x)\rangle\left[\int_{\tau_{-}(x, \xi)}^{0} F\left(\gamma_{x, \xi}(t), \dot{\gamma}_{x, \xi}(t)\right) d t\right] d \Sigma^{2 n-2}(x, \xi)
$$

whenever $F \in C(\Omega M)$.

Taking $F(x, \xi)=f_{i j}(x) \xi^{i} \xi^{j}$, from (3.6) we deduce

$$
\int_{M}\left[\int_{\Omega_{x} M} \xi^{i} \xi^{j} d \omega_{x}(\xi)\right] f_{i j}(x) d V^{n}(x)=\int_{\partial_{+} \Omega M}\langle\xi, \nu(x)\rangle \operatorname{If}(x, \xi) d \Sigma^{2 n-2}(x, \xi) .
$$

In the above and in the rest of the paper $\operatorname{If}(x, \xi)$ is the ray transform applied to the geodesic $\gamma_{x, \xi}$ which is parameterized by arc length on $\left[\tau_{-}(x, \xi), 0\right]$. Proposition 3.1 applies to these geodesic segments by a simple reparametrization.

The left-hand side of this equality is nothing but $(1 / n) \lambda$ with

$$
\lambda=(g, f)_{L_{2}\left(S^{2} \tau_{M}^{\prime}\right)} .
$$

We thus arrive at the formula

$$
\lambda=n \int_{\partial_{+} \Omega M}\langle\xi, \nu\rangle \operatorname{Ifd} \Sigma^{2 n-2} .
$$


Observe that $\lambda=(g, f)_{L_{2}\left(S^{2} \tau_{M}^{\prime}\right)}$ is half of the derivative of the volume of the manifold $\left(M, g^{\tau}\right)$ with respect to $\tau$ at $\tau=0$. So we proceed with studying the volume of $\left(M, g^{\tau}\right)$.

\section{Volume of the Metric $g^{\tau}=g+\tau f$}

The purpose of this section is to prove:

Proposition 4.1. Let $(M, g)$ be a compact Riemannian manifold with boundary. There exists an $\varepsilon>0$ such that if $f \in C\left(S^{2} \tau_{M}^{\prime}\right)$ satisfies

$$
\|f\|_{C\left(S^{2} \tau_{M}^{\prime}\right)}<\varepsilon
$$

and if $\operatorname{Vol}(g+f) \leq \operatorname{Vol}(g)$, then

$$
\lambda=(g, f)_{L_{2}\left(S^{2} \tau_{M}^{\prime}\right)} \leq \frac{2}{3}\|f\|_{L_{2}\left(S^{2} \tau_{M}^{\prime}\right)}^{2} .
$$

Proof. We choose a domain $D \subset \mathbb{R}^{n}$ and a smooth mapping $D \rightarrow M$ that carries $D$ diffeomorphically onto an open set of $M$ whose closure coincides with $M$. Denote the volume of $M$ in the metric $g^{\tau}=g+\tau f$ by $V(\tau)$. Then

$$
V(\tau)=\int_{D}\left(\operatorname{det} g^{\tau}\right)^{1 / 2} d x
$$

We represent the integrand of (4.1) as follows:

$$
\begin{aligned}
& \operatorname{det} g^{\tau}=\operatorname{det}(g+\tau f)=\operatorname{det} g \cdot \operatorname{det}\left(E+\tau g^{-1} f\right) ; \\
& \operatorname{det} g^{\tau}=\operatorname{det} g \cdot\left(1+\lambda_{1} \tau+\lambda_{2} \tau^{2}+\cdots+\lambda_{n} \tau^{n}\right),
\end{aligned}
$$

where $\lambda_{k}$ is the $k$-th elementary symmetric function in the eigenvalues $\mu_{1}, \ldots, \mu_{n}$ of the matrix $g^{-1} f$. The eigenvalues are real. Note that

$$
\langle g, f\rangle=f_{i}^{i}=\lambda_{1}
$$

and

$$
|f|^{2}=f_{i j} f^{i j}=\sum_{k=1}^{n} \mu_{k}^{2}=\lambda_{1}^{2}-2 \lambda_{2} .
$$

Our assumptions and (4.4) imply the estimate

$$
\left|\lambda_{k}\right| \leq C_{k}|f|^{k} \leq C_{k} \varepsilon^{k}
$$

Using the inequality

$$
\sqrt{1+x} \geq 1+\frac{1}{2} x-\frac{1}{4} x^{2} \quad\left(|x| \leq \frac{1}{2}\right),
$$

from (4.2) we obtain

$$
\begin{aligned}
\left(\operatorname{det} g^{\tau}\right)^{1 / 2} \geq & (\operatorname{det} g)^{1 / 2}\left[1+\frac{1}{2}\left(\lambda_{1} \tau+\lambda_{2} \tau^{2}+\cdots+\lambda_{n} \tau^{n}\right)\right. \\
& \left.-\frac{1}{4}\left(\lambda_{1} \tau+\lambda_{2} \tau^{2}+\cdots+\lambda_{n} \tau^{n}\right)^{2}\right] .
\end{aligned}
$$

With the help of (4.5), the last inequality implies the estimate

$$
\left(\operatorname{det} g^{\tau}\right)^{1 / 2} \geq(\operatorname{det} g)^{1 / 2}\left[1+\frac{1}{2} \lambda_{1} \tau+\left(\frac{1}{2} \lambda_{2}-\frac{1}{4} \lambda_{1}^{2}\right) \tau^{2}-C \varepsilon|f|^{2} \tau^{3}\right]
$$


with some constant $C$ depending only on $n$. Expressing $\lambda_{2}$ through $\lambda_{1}$ and $|f|^{2}$ by (4.4) and inserting the resultant expression in the preceding inequality, we obtain

$$
\left(\operatorname{det} g^{\tau}\right)^{1 / 2} \geq(\operatorname{det} g)^{1 / 2}\left[1+\frac{1}{2} \lambda_{1} \tau-\frac{1}{4}|f|^{2} \tau^{2}-C \varepsilon|f|^{2} \tau^{3}\right] .
$$

Integrating this inequality over $D$, we discover that

$$
V(\tau) \geq V(0)+\frac{1}{2} \lambda \tau-\frac{1}{4}\|f\|_{L_{2}}^{2} \tau^{2}-C \varepsilon\|f\|_{L_{2}}^{2} \tau^{3} .
$$

Since $V(1) \leq V(0)$, this inequality implies that

$$
\lambda \leq\left(\frac{1}{2}+C \varepsilon\right)\|f\|_{L_{2}\left(S^{2} \tau_{M}^{\prime}\right)}^{2} .
$$

Choosing an appropriately small $\varepsilon$, we may conclude that

$$
\lambda \leq \frac{2}{3}\|f\|_{L_{2}\left(S^{2} \tau_{M}^{\prime}\right)}^{2} .
$$

\section{LOCAL estimates For If NEAR $\partial_{0} \Omega M$}

On a CDRM $M$, the definition of the ray transform

$$
I f(x, \xi)=\int_{\tau_{-}(x, \xi)}^{0} f_{i j}\left(\gamma_{x, \xi}(t)\right) \dot{\gamma}_{x, \xi}^{i}(t) \dot{\gamma}_{x, \xi}^{j}(t) d t \quad\left((x, \xi) \in \partial_{+} \Omega M\right)
$$

and smoothness of the function $\tau_{-}(x, \xi)$ on $\partial_{+} \Omega M$ (see Lemma 4.1.1 of [Sh2]) imply the boundedness of the ray transform in the $C^{k}$-norms

$$
\|I f\|_{C^{k}\left(\partial_{+} \Omega M\right)} \leq C_{k}\|f\|_{C^{k}\left(S^{2} \tau_{M}^{\prime}\right)} .
$$

The condition that the metrics $g$ and $g+f$ induce the same metric on $\partial M$ is

$$
f_{i j}(x) \xi^{i} \eta^{j}=0 \quad \text { for } \quad x \in \partial M ; \quad \xi, \eta \in T_{x}(\partial M) .
$$

Lemma 5.1. If $M$ is a CDRM and a tensor field $f \in C^{2}\left(S^{2} \tau_{M}^{\prime}\right)$ satisfies (5.3), then the ray transform If vanishes on the boundary $\partial_{0} \Omega M$ of the manifold $\partial_{+} \Omega M$ together with all its first order derivatives.

Proof. In a neighborhood of a point $x_{0} \in \partial M$ we can choose semigeodesic coordinates $\left(x^{1}, \ldots, x^{n}\right)$ such that $\left|x^{n}\right|$ coincides with the distance from $x$ to $\partial M$. In this coordinate system, $g_{i n}=\delta_{i n}$ and the Christoffel symbols satisfy the relations

$$
\Gamma_{n n}^{i}=\Gamma_{i n}^{n}=0, \quad \Gamma_{\beta n}^{\alpha}=-g^{\alpha \gamma} \Gamma_{\beta \gamma}^{n} .
$$

(In this and subsequent formulas, Greek indices vary from 1 to $n-1$; and repeated Greek indices imply the summation from 1 to $n-1$ as usual). The outward unit normal vector $\nu$ to $\partial M$ has coordinates $(0, \ldots, 0,1)$, and $\langle\xi, \nu\rangle=\xi^{n}=\xi_{n}$. The second fundamental form of $\partial M$

$$
\mathrm{II}(\xi, \xi)=-\Gamma_{\alpha \beta}^{n}\left(x^{1}, \ldots, x^{n-1}, 0\right) \xi^{\alpha} \xi^{\beta}
$$

is positive definite because of the strict convexity of the boundary. Condition (5.3) is written in the chosen coordinates as

$$
\left.f_{\alpha \beta}\right|_{x^{n}=0}=0 .
$$

Let $\left(x^{1}, \ldots, x^{n} ; \xi^{1}, \ldots, \xi^{n}\right)$ be the associated coordinate system on $T M$. Then $\left(x^{1}, \ldots, x^{n-1} ; \xi^{1}, \ldots, \xi^{n}\right)$ constitute a local coordinate system on $\partial(T M)$. The 
submanifold $\partial_{+} \Omega M$ of $\partial(T M)$ is determined in these coordinates by the relations $g_{i j}(x) \xi^{i} \xi^{j}=1, \xi^{n}=\xi_{n} \geq 0$; and its boundary $\partial_{0} \Omega M$ is determined by $g_{\alpha \beta}(x) \xi^{\alpha} \xi^{\beta}=1, \xi^{n}=0$.

The equality

$$
\left.\tau_{-}(x, \xi)\right|_{\xi_{n}=0}=0 \quad((x, \xi) \in \partial(T M))
$$

is evident. We will now find the derivative $\left.\frac{\partial \tau_{-}(x, \xi)}{\partial \xi_{n}}\right|_{\xi_{n}=0}$. To this end, given a point $(x, \xi) \in \partial(T M), \xi \neq 0$, we denote by

$$
\gamma(t ; x, \xi)=\left(\gamma^{1}(t ; x, \xi), \ldots, \gamma^{n}(t ; x, \xi)\right)
$$

the geodesic in $M$ that satisfies the initial conditions

$$
\gamma^{\alpha}(0 ; x, \xi)=x^{\alpha}, \quad \gamma^{n}(0 ; x, \xi)=0, \quad \dot{\gamma}^{i}(0 ; x, \xi)=\xi^{i} .
$$

The equation

$$
\gamma^{n}(t ; x, \xi)=0
$$

has two solutions $t=0$ and $t=\tau_{-}(x, \xi)$. Representing the function $\gamma^{n}(t ; x, \xi)$ in the form

$$
\gamma^{n}(t ; x, \xi)=\xi_{n} t-\frac{1}{2} \Gamma_{\alpha \beta}^{n}(x) \xi^{\alpha} \xi^{\beta} t^{2}+\varphi(t ; x, \xi) t^{3}
$$

with some smooth function $\varphi(t ; x, \xi)$, we see that $\tau_{-}(x, \xi)$ satisfies the equation

$$
\xi_{n}-\frac{1}{2} \Gamma_{\alpha \beta}^{n}(x) \xi^{\alpha} \xi^{\beta} \tau_{-}(x, \xi)+\varphi\left(\tau_{-}(x, \xi) ; x, \xi\right) \tau_{-}^{2}(x, \xi)=0 .
$$

Differentiating this equation with respect to $\xi_{n}$ and putting $\xi_{n}=0$, we obtain

$$
\left.\frac{\partial \tau_{-}(x, \xi)}{\partial \xi_{n}}\right|_{\xi_{n}=0}=2\left(\Gamma_{\alpha \beta}^{n}(x) \xi^{\alpha} \xi^{\beta}\right)^{-1} .
$$

We have to prove that

$$
\left.I f\right|_{\xi_{n}=0}=0,\left.\quad \frac{\partial(I f)}{\partial \xi_{n}}\right|_{\xi_{n}=0}=0 .
$$

The first of these equalities follows from definition (5.1) and (5.5). To prove the second one, we rewrite (5.1) in the form

$$
I f(x, \xi)=\int_{\tau_{-}(x, \xi)}^{0} F(t ; x, \xi) d t
$$

where

$$
F(t ; x, \xi)=f_{i j}(\gamma(t ; x, \xi)) \dot{\gamma}^{i}(t ; x, \xi) \dot{\gamma}^{j}(t ; x, \xi) .
$$

Differentiating (5.8), we obtain

$$
\frac{\partial(I f)}{\partial \xi_{n}}=-\frac{\partial \tau_{-}}{\partial \xi_{n}} F\left(\tau_{-}(x, \xi) ; x, \xi\right)+\int_{\tau_{-}(x, \xi)}^{0} \frac{\partial F(t ; x, \xi)}{\partial \xi_{n}} d t .
$$

Putting $\xi_{n}=0$ in this formula and using (5.5), we derive

$$
\left.\frac{\partial(I f)}{\partial \xi_{n}}\right|_{\xi_{n}=0}=\left[-\frac{\partial \tau_{-}}{\partial \xi_{n}} F\right]_{t=0, \xi_{n}=0} .
$$


In view of (5.4), equality (5.9) implies

$$
\left.F\right|_{t=0, \xi_{n}=0}=f_{\alpha \beta}(x) \xi^{\alpha} \xi^{\beta}=0 .
$$

This relation together with (5.10) implies the second of equalities (5.7). The lemma is proved.

Corollary 5.2. Let

$$
L: C^{\infty}\left(\partial_{+} \Omega M\right) \rightarrow C^{\infty}\left(\partial_{+} \Omega M\right)
$$

be a first order linear differential operator with smooth coefficients on the manifold $\partial_{+} \Omega M$. If $f \in C^{2}\left(S^{2} \tau_{M}^{\prime}\right)$ is a tensor field satisfying (5.3), then the estimate

$$
|L(I f)(x, \xi)| \leq C\langle\xi, \nu(x)\rangle\|f\|_{C^{2}\left(S^{2} \tau_{M}^{\prime}\right)}
$$

holds with some constant $C$ independent of $f$.

Proof. For $\left(x, \xi_{1}\right) \in \partial_{+} \Omega M$, we can choose a curve $t \mapsto \xi_{t}, 0 \leq t \leq 1$, in the sphere $\Omega_{x} M$ which joins $\xi_{1}$ with a point $\xi_{0}$ such that

$$
\left\langle\xi_{0}, \nu(x)\right\rangle=0, \quad\left\langle\xi_{t}, \nu(x)\right\rangle \geq 0, \quad\left|\frac{d \xi_{t}}{d t}\right|=\frac{\pi}{2}\left\langle\xi_{1}, \nu(x)\right\rangle .
$$

By Lemma $5.1, L(I f)\left(x, \xi_{0}\right)=0$. Therefore,

$$
L(I f)\left(x, \xi_{1}\right)=\int_{0}^{1} \frac{d}{d t}\left[L(I f)\left(x, \xi_{t}\right)\right] d t .
$$

The integral in this formula admits the estimate

$$
\left|L(I f)\left(x, \xi_{1}\right)\right| \leq \int_{0}^{1}\left|\operatorname{grad}(L(I f))\left(x, \xi_{t}\right)\right|\left|\frac{d \xi_{t}}{d t}\right| d t \leq C\|I f\|_{C^{2}} \cdot\left\langle\xi_{1}, \nu(x)\right\rangle
$$

which, together with (5.2), gives (5.11).

Lemma 5.3. Let $M$ be a $C D R M$ and a tensor field $f \in C^{2}\left(S^{2} \tau_{M}^{\prime}\right)$ satisfy (5.3). Fix a semigeodesic coordinate system $\left(x^{1}, \ldots, x^{n}\right)$ in a neighborhood $U$ of a point $x_{0} \in \partial M$ such that $\left|x^{n}\right|=\operatorname{dist}(x, \partial M)$. Then the inequality

$$
\mid \xi^{\alpha} \stackrel{v}{\nabla}_{n} \stackrel{h}{\nabla}_{\alpha}(I f)(x, \xi)<C\|f\|_{C^{2}} \cdot\langle\xi, \nu(x)\rangle
$$

holds for all $x \in U \cap \partial M$ with a constant $C$ independent of $f$. Here the summation from 1 to $n-1$ is meant with respect to the index $\alpha$.

Proof. The left-hand side of (5.12) can be written as follows:

$$
\xi^{\alpha} \stackrel{v}{\nabla} \stackrel{h}{\nabla}_{\alpha}(I f)=\xi^{\alpha} \frac{\partial^{2}(I f)}{\partial x^{\alpha} \partial \xi^{n}}+L(I f)
$$

where $L$ is a first order linear differential operator on $\partial_{+} \Omega M$. On taking Corollary 5.2 into account, estimate (5.12) follows from the inequality

$$
\left|\frac{\partial^{2} I f(x, \xi)}{\partial x^{\alpha} \partial \xi^{n}}\right| \leq C\langle\xi, \nu(x)\rangle\|f\|_{C^{2}} .
$$

So our goal is proving estimate (5.13). 
Differentiating (5.8), we obtain

$$
\begin{aligned}
\frac{\partial^{2}(I f(x, \xi))}{\partial x^{\alpha} \partial \xi^{n}}= & -\frac{\partial^{2} \tau_{-}(x, \xi)}{\partial x^{\alpha} \partial \xi^{n}} F\left(\tau_{-}(x, \xi) ; x, \xi\right) \\
& -\frac{\partial \tau_{-}(x, \xi)}{\partial x^{\alpha}} \frac{\partial}{\partial \xi^{n}}\left[F\left(\tau_{-}(x, \xi) ; x, \xi\right)\right] \\
& -\frac{\partial \tau_{-}(x, \xi)}{\partial \xi^{n}} \frac{\partial F}{\partial x^{\alpha}}\left(\tau_{-}(x, \xi) ; x, \xi\right)+\int_{\tau_{-}(x, \xi)}^{0} \frac{\partial^{2} F(t ; x, \xi)}{\partial x^{\alpha} \partial \xi^{n}} d t
\end{aligned}
$$

Introducing the notation

$$
\varphi(x, \xi)=F\left(\tau_{-}(x, \xi) ; x, \xi\right)
$$

we have

$$
\frac{\partial F}{\partial x^{\alpha}}\left(\tau_{-}(x, \xi) ; x, \xi\right)=\frac{\partial \varphi(x, \xi)}{\partial x^{\alpha}}-\frac{\partial F}{\partial t}\left(\tau_{-}(x, \xi) ; x, \xi\right) \frac{\partial \tau_{-}(x, \xi)}{\partial x^{\alpha}} .
$$

Substituting this expression into (5.14), we obtain

$$
\begin{aligned}
\frac{\partial^{2}(I f(x, \xi))}{\partial x^{\alpha} \partial \xi^{n}}= & -\frac{\partial^{2} \tau_{-}(x, \xi)}{\partial x^{\alpha} \partial \xi^{n}} \varphi(x, \xi) \\
& -\frac{\partial \tau_{-}(x, \xi)}{\partial x^{\alpha}}\left(\frac{\partial \varphi(x, \xi)}{\partial \xi^{n}}+\frac{\partial F}{\partial t}\left(\tau_{-}(x, \xi) ; x, \xi\right)\right) \\
& -\frac{\partial \tau_{-}(x, \xi)}{\partial \xi^{n}} \frac{\partial \varphi(x, \xi)}{\partial x^{\alpha}}+\int_{\tau_{-}(x, \xi)}^{0} \frac{\partial^{2} F(t ; x, \xi)}{\partial x^{\alpha} \partial \xi^{n}} d t .
\end{aligned}
$$

Formulas (5.9) and (5.15) imply the estimates

$$
\|F\|_{C^{k}} \leq C\|f\|_{C^{k}}, \quad\|\varphi\|_{C^{k}} \leq C\|f\|_{C^{k}}
$$

for every $k$. Therefore (5.16) implies the inequality

$$
\begin{aligned}
& \left|\frac{\partial^{2} I f(x, \xi)}{\partial x^{\alpha} \partial \xi^{n}}\right| \\
& \quad \leq C\left(|\varphi(x, \xi)|+\left|\frac{\partial \varphi(x, \xi)}{\partial x^{\alpha}}\right|+\left|\frac{\partial \tau_{-}(x, \xi)}{\partial x^{\alpha}}\right|\|f\|_{C^{1}}+\left|\tau_{-}(x, \xi)\right| \cdot\|f\|_{C^{2}}\right) .
\end{aligned}
$$

The latter inequality would imply estimate (5.13) if we demonstrate that

$$
\begin{gathered}
\left|\tau_{-}(x, \xi)\right| \leq C\langle\xi, \nu(x)\rangle, \quad \frac{\partial \tau_{-}(x, \xi)}{\partial x^{\alpha}} \leq C\langle\xi, \nu(x)\rangle, \\
|\varphi(x, \xi)| \leq C\langle\xi, \nu(x)\rangle\|f\|_{C^{1}}, \quad\left|\frac{\partial \varphi(x, \xi)}{\partial x^{\alpha}}\right| \leq C\langle\xi, \nu(x)\rangle\|f\|_{C^{2}} .
\end{gathered}
$$

Estimates (5.17) are evident because $\tau_{-}(x, \xi)$ and $\partial \tau_{-}(x . \xi) / \partial x^{\alpha}$ are smooth functions on $\partial_{+} \Omega M$ vanishing on the boundary $\partial_{0} \Omega M$ which is determined by the equation $\langle\xi, \nu(x)\rangle=0$.

To prove estimates (5.18) we first note that the function $\varphi(x, \xi)$ (and, consequently, $\left.\partial \varphi(x, \xi) / \partial x^{\alpha}\right)$ vanishes on $\partial_{0} \Omega M$. Indeed, $\tau_{-}(x, \xi)=0$ for $(x, \xi) \in \partial_{0} \Omega M$, and definitions (5.9) and (5.15) give us

$$
\varphi(x, \xi)=f_{i j}(x) \xi^{i} \xi^{j} .
$$

Since $f_{\alpha \beta}(x)=0(1 \leq \alpha, \beta \leq n-1)$ and $\xi^{n}=0$, this implies that $\varphi(x, \xi)=0$. 
Given a point $\left(x, \xi_{1}\right) \in \partial_{+} \Omega M$, we can join it with a point $\left(x, \xi_{0}\right) \in \partial_{0} \Omega M$ by a curve $\left(x, \xi_{t}\right) \in \partial_{+} \Omega M(0 \leq t \leq 1)$ such that $\left|d \xi_{t} / d t\right|=\left\langle\xi_{1}, \nu(x)\right\rangle$. Using the representations

$$
\varphi\left(x, \xi_{1}\right)=\int_{0}^{1} \frac{d}{d t}\left(\varphi\left(x, \xi_{t}\right)\right) d t, \quad \frac{\partial \varphi\left(x, \xi_{1}\right)}{\partial x^{\alpha}}=\int_{0}^{1} \frac{d}{d t}\left(\frac{\partial \varphi}{\partial x^{\alpha}}\left(x, \xi_{t}\right)\right) d t
$$

we obtain the estimates

$$
\begin{aligned}
& \left|\varphi\left(x, \xi_{1}\right)\right| \leq \int_{0}^{1}\|\varphi\|_{C^{1}}\left|\frac{d \xi_{t}}{d t}\right| d t \leq C\left\langle\xi_{1}, \nu(x)\right\rangle\|f\|_{C^{1}}, \\
& \left|\frac{\partial \varphi\left(x, \xi_{1}\right)}{\partial x^{\alpha}}\right| \leq \int_{0}^{1}\|\varphi\|_{C^{2}}\left|\frac{d \xi_{t}}{d t}\right| d t \leq C\left\langle\xi_{1}, \nu(x)\right\rangle\|f\|_{C^{2}}
\end{aligned}
$$

that are equivalent to (5.18). The lemma is proved.

\section{Proof of TheOrem 1.1}

To prove the main theorem, it is sufficient to prove Proposition 1.2. Thus we let $(M, g)$ satisfy the hypotheses of Proposition 1.2 and let $g^{1}$ be a metric $C^{3, \alpha_{-}}$ close enough to $g$ and such that the boundary distance-functions satisfy $d_{g^{1}}(x, y) \geq$ $d_{g}(x, y)$ for all $x, y \in \partial M$, the induced Riemannian metrics on $\partial M$ coincide, and $\operatorname{Vol}\left(g_{1}\right) \leq \operatorname{Vol}(g)$. We will show that $g^{1}$ is isometric to $g$. In view of Theorem 2.1, we may assume that the tensor field $f=g^{1}-g$ is solenoidal and satisfies the inequality $\|f\|_{C^{2}\left(S^{2} \tau_{M}^{\prime}\right)}<\varepsilon$ with an arbitrary small $\varepsilon>0$. By choosing $\varepsilon$ sufficiently small and applying Proposition 3.1, equation (3.7), and Proposition 4.1 we see that the tensor field $f$ satisfies

$$
\begin{gathered}
\delta f=0 \\
\|f\|_{C^{2}\left(S^{2} \tau_{M}^{\prime}\right)}<\varepsilon \\
I f \geq 0 \\
n \int_{\partial_{+} \Omega M}\langle\xi, \nu\rangle I f d \Sigma^{2 n-2}=\lambda \leq \frac{2}{3}\|f\|_{L_{2}\left(S^{2} \tau_{M}^{\prime}\right)}^{2} .
\end{gathered}
$$

We will prove that $f=0$.

Given $f$, we define the function $u \in C^{2}\left(T^{0} M \backslash T(\partial M)\right)$ by the equality

$$
u(x, \xi)=\int_{\tau_{-}(x, \xi)}^{0} f_{i j}\left(\gamma_{x, \xi}(t)\right) \dot{\gamma}_{x, \xi}^{i}(t) \dot{\gamma}_{x, \xi}^{j}(t) d t \quad\left((x, \xi) \in T^{0} M\right),
$$

where $T^{0} M=\{(x, \xi) \in T M \mid \xi \neq 0\}$ is the manifold of nonzero tangent vectors. This function satisfies the boundary conditions

$$
\left.u\right|_{\partial_{-} \Omega M}=0
$$


and

$$
u(x, \xi)=I f(x, \xi) \geq 0 \quad \text { for } \quad(x, \xi) \in \partial_{+} \Omega M .
$$

The inequality (6.6) is just (6.3).

Since $f$ is solenoidal, the Pestov integral identity for the function $u$ (formula (4.6.13) of [Sh2]) is:

$$
\begin{gathered}
\int_{\Omega M}\left[|\stackrel{h}{\nabla} u|^{2}-R_{i j k l} \xi^{i} \xi^{k} \nabla^{v} u \cdot \nabla^{l} u+(n+2)|H u|^{2}\right] d \Sigma \\
=\int_{\partial_{+} \Omega M}\left[L(I f)-4(I f) f_{i j} \xi^{i} \nu^{j}\right] d \Sigma^{2 n-2},
\end{gathered}
$$

where $H$ is the vector field on $\Omega M$ that generates the geodesic flow, and $L$ is the quadratic first order differential operator on the manifold $\partial_{+} \Omega M$ which is expressed in semigeodesic coordinates $\left(x^{1}, \ldots, x^{n-1}, x^{n}=\right.$ distance to the boundary) as follows (formula (4.6.14) of [Sh2]):

$$
L u=\xi^{n} \stackrel{h}{\nabla} u \cdot \stackrel{v}{\nabla}_{\alpha} u-\xi^{\alpha} \stackrel{h}{\nabla}_{\alpha} u \cdot \stackrel{v}{\nabla}^{n} u .
$$

The heart of the rest of the proof is:

Lemma 6.1. There is a constant $C$ independent of $f$ and such that

$$
\int_{\partial_{+} \Omega M}\left[L(I f)-4(I f) f_{i j} \xi^{i} \nu^{j}\right] d \Sigma^{2 n-2} \leq C\|f\|_{C^{2}\left(S^{2} \tau_{M}^{\prime}\right)} \lambda .
$$

We will come back to the proof of this lemma but we first show how the theorem will follow.

Lemma 6.1, along with (6.7), gives

$$
\int_{\Omega M}\left[\left|\frac{h}{\nabla} u\right|^{2}+(n+2)|H u|^{2}\right] d \Sigma \leq \int_{\Omega M} R_{i j k l} \xi^{i} \xi^{k} \nabla^{v} u \cdot \nabla^{l} u d \Sigma+C\|f\|_{C^{2}} \lambda .
$$

The integral on the right-hand side of (6.9) can be estimated by formula (4.7.8) of Sh2:

$$
\int_{\Omega M} R_{i j k l} \xi^{i} \xi^{k} \nabla^{v} u \cdot \nabla^{l} u d \Sigma \leq 3 k^{+}(n+2) \int_{\Omega M}|H u|^{2} d \Sigma+3 k^{+} \int_{\Omega M}\left|{ }^{h} u\right|^{2} d \Sigma,
$$

where $k^{+}=k^{+}(M, g)$ is defined by (1.1). With the help of this inequality, (6.9) gives

$$
\left(1-3 k^{+}\right)\left(\int_{\Omega M}\left|\frac{h}{\nabla} u\right|^{2} d \Sigma+(n+2) \int_{\Omega M}|H u|^{2} d \Sigma\right) \leq C\|f\|_{C^{2}} \lambda .
$$

Using (6.2) and (6.4), we obtain

$$
\left(1-3 k^{+}\right)\left(\int_{\Omega M}|\nabla u|^{2} d \Sigma+(n+2) \int_{\Omega M}|H u|^{2} d \Sigma\right) \leq C \varepsilon\|f\|_{L_{2}}^{2} .
$$


Finally, the kinetic equation $H u=f_{i j}(x) \xi^{i} \xi^{j}$ implies the estimate

$$
\|f\|_{L_{2}}^{2} \leq C^{\prime} \int_{\Omega M}|H u|^{2} d \Sigma
$$

with some constant $C^{\prime}$ independent of $f$. Combining (6.10) and (6.11), we arrive at the final estimate

$$
\left(1-3 k^{+}\right) \int_{\Omega M}\left|\nabla^{h} u\right|^{2} d \Sigma+\left[(n+2)\left(1-3 k_{+}\right)-C C^{\prime} \varepsilon\right] \int_{\Omega M}|H u|^{2} d \Sigma \leq 0 .
$$

Since we can choose $\varepsilon>0$ arbitrarily small, we can choose it so that the coefficients of both integrals in (6.12) are positive. Therefore (6.12) implies that $H u \equiv 0$ and hence $f \equiv 0$. The theorem is proved.

Proof of Lemma 6.1. First we transform the integral $\int_{\partial_{+} \Omega M} L u d \Sigma^{2 n-2}$ by integration by parts. To this end we rewrite (6.8) as follows:

$$
L u=a^{i} \stackrel{v}{\nabla}_{i} u
$$

where

$$
a^{\alpha}=\xi^{n} \nabla^{\alpha} u, \quad a^{n}=-\xi^{\alpha} \stackrel{h}{\alpha}_{\alpha} u
$$

We extract a divergent term from (6.13):

$$
L u=\stackrel{v}{\nabla}_{i}\left(u a^{i}\right)-u \stackrel{v}{\nabla} a_{i}^{i}=\stackrel{v}{\nabla}_{i}\left(u a^{i}\right)-\xi^{n} u \stackrel{v}{\nabla} \stackrel{h}{\nabla}^{\alpha} u+u \stackrel{v}{\nabla}\left(\xi^{\alpha} \stackrel{h}{\nabla}, u\right) .
$$

Integrating this equality over $\partial_{+} \Omega M$ and transforming the first term by Gauss Ostrogradskiu, we obtain

$$
\begin{gathered}
\int_{\partial_{+} \Omega M} L u d \Sigma^{2 n-2}=k \int_{\partial_{+} \Omega M} u\langle\xi, a\rangle d \Sigma^{2 n-2} \\
-\int_{\partial_{+} \Omega M}\left[\langle\xi, \nu\rangle u \stackrel{v}{\nabla_{\alpha}} \stackrel{h}{\nabla}^{\alpha} u-u \stackrel{v}{\nabla}_{n}\left(\xi^{\alpha} \stackrel{h}{\nabla}_{\alpha} u\right)\right] d \Sigma^{2 n-2} .
\end{gathered}
$$

The coefficient $k$ depends on the degree of homogeneity of $a$. Its value does not matter because $\langle\xi, a\rangle=0$ as we see from (6.14). Consequently,

$$
\int_{\partial_{+} \Omega M} L u d \Sigma^{2 n-2}=-\int_{\partial_{+} \Omega M}\left[\langle\xi, \nu\rangle u \stackrel{v}{\nabla_{\alpha}} \stackrel{h}{\nabla}^{\alpha} u-u \xi^{\alpha} \nabla_{n}^{v} \stackrel{h}{\alpha}_{\alpha} u\right] d \Sigma^{2 n-2} .
$$

We thus see that

$$
\begin{gathered}
\int_{\partial_{+} \Omega M}\left[L(I f)-4(I f) f_{i j} \xi^{i} \nu^{j}\right] d \Sigma^{2 n-2} \\
=-\int_{\partial_{+} \Omega M}\langle\xi, \nu\rangle I f \cdot \nabla_{\alpha} \stackrel{h}{ }^{\alpha}(I f) d \Sigma^{2 n-2} \\
+\int_{\partial_{+} \Omega M} I f \cdot \xi^{\alpha} \stackrel{v}{\nabla}_{n}^{h} \stackrel{h}{\alpha}_{\alpha}(I f) d \Sigma^{2 n-2}-4 \int_{\partial_{+} \Omega M} I f \cdot f_{i j} \xi^{i} \nu^{j} d \Sigma^{2 n-2} .
\end{gathered}
$$


Some terms in this equation are written by using local coordinates. Nevertheless, all the integrands are invariant; i.e., they are independent of the choice of coordinates.

We will now estimate each of the integrals on the right-hand side of (6.15).

Using the nonnegativity of $I f$, we obtain

$$
\left|\int_{\partial_{+} \Omega M}\langle\xi, \nu\rangle I f \cdot \stackrel{v}{\nabla}_{\alpha} \nabla^{\alpha}(I f) d \Sigma^{2 n-2}\right| \leq\|I f\|_{C^{2}} \int_{\partial_{+} \Omega M}\langle\xi, \nu\rangle I f d \Sigma^{2 n-2} .
$$

Together with (6.4) and (5.2), this gives

$$
\left|\int_{\partial_{+} \Omega M}\langle\xi, \nu\rangle I f \cdot \stackrel{v}{\nabla}_{\alpha}^{h} \nabla^{\alpha}(I f) d \Sigma^{2 n-2}\right| \leq C\|f\|_{C^{2} \lambda}
$$

with some constant $C$ independent of $f$.

Applying Lemma 5.3, we obtain

$$
\left|\int_{\partial_{+} \Omega M} I f \cdot \xi^{\alpha} v_{n}^{v} \stackrel{h}{\nabla}_{\alpha}(I f) d \Sigma^{2 n-2}\right| \leq C\|f\|_{C^{2}} \int_{\partial_{+} \Omega M}\langle\xi, \nu\rangle I f d \Sigma^{2 n-2}=C\|f\|_{C^{2}} \lambda .
$$

The estimation of the last integral on the right-hand side of (6.15),

$$
J=\int_{\partial_{+} \Omega M} I f \cdot f_{i j} \xi^{i} \nu^{j} d \Sigma^{2 n-2}
$$

is more troublesome because the factor $f_{i j} \xi^{i} \nu^{j}$ of its integrand does not vanish on $\partial_{0} \Omega M$ (more precisely, we are not able to prove that it vanishes a priori). To estimate this integral, we introduce the mapping

$$
\Phi: \partial_{+} \Omega M \rightarrow \partial_{+} \Omega M
$$

by putting

$$
\Phi(x, \xi)=(y, \eta), \quad \text { where } \quad y=\gamma_{x, \xi}\left(\tau_{-}(x, \xi)\right), \quad \eta=-\dot{\gamma}_{x, \xi}\left(\tau_{-}(x, \xi)\right) .
$$

It is evident that $\Phi$ is smooth and $\Phi^{2}=$ Id. Consequently, $\Phi$ is a diffeomorphism. One can see (Lemma [Cr3] ) by a double use of Santaló's formula and the fact that the map $v \mapsto-v$ is measure preserving on $\Omega M$ that the absolute value of the Jacobian on $\partial_{+} \Omega M \backslash \partial_{0} \Omega M$ of $\Phi$ is $\frac{\langle\xi, \nu(x)\rangle}{\langle\eta, \nu(y)\rangle} ;$ i.e.,

$$
\frac{\langle\xi, \nu(x)\rangle}{\langle\eta, \nu(y)\rangle}=\left|\frac{d \Sigma^{2 n-2}(y, \eta)}{d \Sigma^{2 n-2}(x, \xi)}\right| .
$$

We need to study what happens on the boundary $\partial_{0} \Omega M$ of $\partial_{+} \Omega M$. The relations

$$
y(x, \xi)=x, \quad \eta(x, \xi)=-\xi \quad \text { for } \quad(x, \xi) \in \partial_{0} \Omega M
$$

are evident. We will use the same semigeodesic coordinates as in the proof of Lemma 5.1. Early in the proof of Lemma 5.1 we saw

$$
\tau_{-}=0, \quad \frac{\partial \tau_{-}}{\partial \xi^{n}}=2\left(\Gamma_{\alpha \beta}^{n} \xi^{\alpha} \xi^{\beta}\right)^{-1}
$$


hold on $\partial_{0} \Omega M$. Differentiating (6.20) and using (6.23), we obtain on $\partial_{0} \Omega M$

$$
\frac{\partial \eta^{n}}{\partial \xi^{n}}=-1-\ddot{\gamma}^{n} \frac{\partial \tau_{-}}{\partial \xi^{n}}=-1+2 \Gamma_{\alpha \beta}^{n} \xi^{\alpha} \xi^{\beta}\left(\Gamma_{\alpha \beta}^{n} \xi^{\alpha} \xi^{\beta}\right)^{-1}=1 .
$$

Since $\langle\xi, \nu\rangle=\xi^{n}$ and $\langle\eta, \nu\rangle=\eta^{n}$ in the chosen coordinates, the latter relation implies the representation

$$
\langle\eta, \nu\rangle=\frac{\langle\xi, \nu\rangle}{1+\langle\xi, \nu\rangle \varphi}
$$

with some $\varphi \in C^{\infty}\left(\partial_{+} \Omega M\right)$. In particular, this means that the absolute value of the Jacobian of $\Phi$ (i.e. $\left.\frac{\langle\xi, \nu\rangle}{\langle\eta, \nu\rangle}\right)$ goes to 1 as $(x, \xi)$ approaches $\partial_{0} \Omega M$.

We observe that the ray transform is invariant under $\Phi$, i.e.,

$$
I f(y, \eta)=I f(x, \xi) \quad \text { for } \quad(y, \eta)=\Phi(x, \xi) .
$$

Using the change of variables $(y, \eta)=\Phi(x, \xi)$ in (6.18), we obtain

$$
\begin{gathered}
J=\int_{\partial_{+} \Omega M}(I f)(y, \eta) \cdot f_{i j}(y) \eta^{i} \nu^{j}(y) d \Sigma^{2 n-2}(y, \eta) \\
=\int_{\partial_{+} \Omega M}(I f)(x, \xi) \cdot f_{i j}(y(x, \xi)) \eta^{i}(x, \xi) \nu^{j}(y(x, \xi)) \frac{\langle\xi, \nu\rangle}{\langle\eta, \nu\rangle} d \Sigma^{2 n-2}(x, \xi) .
\end{gathered}
$$

Adding this equality to (6.18), we obtain

$$
\begin{array}{r}
2 J=\int_{\partial_{+} \Omega M}(I f)(x, \xi)\langle\xi, \nu\rangle\left[\frac{f_{i j}(y(x, \xi)) \eta^{i}(x, \xi) \nu^{j}(y(x, \xi))}{\langle\eta, \nu\rangle}\right. \\
\left.+\frac{f_{i j}(x) \xi^{i} \nu^{j}(x)}{\langle\xi, \nu\rangle}\right] d \Sigma^{2 n-2}(x, \xi) .
\end{array}
$$

We now need to bound the expression in brackets from above. We rewrite it as the sum of two terms:

$$
2 J=\int_{\partial_{+} \Omega M}(I f)(x, \xi)\langle\xi, \nu\rangle(A+B) d \Sigma^{2 n-2}(x, \xi),
$$

where

$$
A=\frac{f_{i j}(y(x, \xi)) \eta^{i}(x, \xi) \nu^{j}(y(x, \xi))+f_{i j}(x) \xi^{i} \nu^{j}(x)}{\langle\eta, \nu\rangle}
$$

and

$$
B=f_{i j}(x) \xi^{i} \nu^{j}(x)\left[\frac{1}{\langle\xi, \nu\rangle}-\frac{1}{\langle\eta, \nu\rangle}\right] .
$$

Equations (6.24) and the compactness of $M$ tell us that the part in brackets of $B$ is uniformly bounded by a constant independent of $f$. So $B$ is bounded by a constant times $\|f\|_{C^{0}}$. 
Away from $\partial_{0} \Omega M$ (i.e. $\langle\xi, \nu\rangle \geq$ constant $\left.>0\right) A$ is clearly bounded by a constant times $\|f\|_{C^{0}}$. Near the boundary the numerator of $A$ is bounded by $\|\nabla f\| \rho(x, y(x, \xi))+\|f\|_{C^{0}}|\eta+\xi|$ (where we interpret $\eta+\xi$ as the vector at $x$ with coordinates $\left.\left(\xi^{i}+\eta^{i}\right)\right)$. Now by Lemma 4.1.2 of [Sh2], $\rho(x, y(x, \xi))=\left|\tau_{-}(x, \xi)\right| \leq$ constant $\langle\xi, \nu(x)\rangle$ and hence the relations (6.24) tell us that near the boundary $A$ is bounded by a constant times $\|f\|_{C^{1}}$. Thus we see that $A$ is bounded on all of $\partial_{+} \Omega M$ by a constant times $\|f\|_{C^{1}}$.

Combining these estimates with equation (6.26) we get

$$
|J| \leq C\|f\|_{C^{1}} \int_{\partial_{+} \Omega M}\langle\xi, \nu\rangle \operatorname{If} d \Sigma^{2 n-2}=C\|f\|_{C^{1}} \lambda .
$$

Lemma 6.1 (and hence Theorem 1.1) now follows by combining (6.16), (6.17) and (6.27).

Added in Proof. The hypothesis $K^{+}(M, g) \leq 1 / 3$ of the main theorem can be improved to $K^{+}(M, g)<(n+3) /(2 n+4)$ where $n=\operatorname{dim} M$, by a more careful estimation of the curvature dependent of the Pestov identity.

\section{REFERENCES}

[ADN] S. Agmon, A. Douglis, and L. Nirenberg, Estimates near the boundary for solutions of elliptic partial differential equations satisfying general boundary conditions. II, Comm. Pure Appl. Math. 17 (1964), 35-92. MR 28:5252

[An] M. Anderson, Remarks on the compactness of isospectral sets in low dimensions, Duke Math. J. 63 (1991), 699-711. MR 92m:58140]

[BPP] R. Brooks, P. Perry, and P. Petersen, Compactness and finiteness theorems for isospectral manifolds, J. Reine Angew. Math. 426 (1992), 67-89. MR 93f:53034

[Cr1] C. B. Croke, Rigidity and the distance between boundary points, J. Differential Geometry 33 (1991), 445-464. MR 92a:53053

[Cr2] C. B. Croke, Rigidity for surfaces of non-positive curvature, Comment. Math. Helv. 65 (1990), 150-169. MR 91d:53056

[Cr3] C. B. Croke, A sharp four-dimensional isoperimetric inequality, Comment. Math. Helv. 59 (1984), 187-192. MR 83f:53060

[Cr-Sh] C. B. Croke, and V. A. Sharafutdinov, Spectral rigidity of a compact negatively curved manifold, Topology 37 (6) (1998), 1265-1273. MR 99e:58191

[Gr] M. Gromov, Filling Riemannian manifolds, J. Differential Geometry 18 (1983), 1-147. MR 85h:53029

[GK] V. Guillemin and D. Kazhdan, Some inverse spectral results for negatively curved $n$ manifolds, Proc. Sympos. Pure Math., Amer. Math. Soc., Providence, RI (1980), 153-180. MR 81i:58048

[KA] L. V. Kantorovich and G. P. Akilov, Functional analysis (Translated from the Russian by Howard L. Silcock. Second edition), Pergamon Press, Oxford-Elmsford, N.Y., 1982. MR 83h:46002

[Ml] R. Michel, Sur la rigidité imposée par lar longueur des géodésiques, Invent Math. 65 (1981), 71-83. MR 83d:58021

[Ot] J.-P. Otal, Sur les longueur des géodésiques d'une métrique à courbure négative dans le disque, Comment. Math. Helv. 65 (1990), 334-347. MR 91i:53054]

[Sh1] V. A. Sharafutdinov, Integral geometry of a tensor field on a manifold whose curvature is bounded above, Siberian Math. J. 33 (3) (1992), 524-533. MR 94d:53116

[Sh2] V. A. Sharafutdinov, Integral Geometry of Tensor Fields, VSP, Utrecht, the Netherlands, 1994. MR 97h:53077

[Sh3] V. A. Sharafutdinov, Some questions of integral geometry on Anosov manifolds, to appear. 
[Sl] L. Santaló, Integral Geometry and Geometric Probability (Encyclopedia of Mathematics and its Applications. Vol. 1), Addison-Wesley Publishing Co., Reading, Mass.-LondonAmsterdam, 1976. MR 55:6340

[SU] P. Stefanov and G. Uhlmann, Rigidity for metrics with the same lengths of geodesics, Mathematical Research Letters 5 (1998), 83-96. MR 99c:53059

Department of Mathematics, University of Pennsylvania, Philadelphia, Pennsylvania 19104

E-mail address: ccroke@math.upenn.edu

Sobolev Institute of Mathematics, Universitetskil PR., 4, Novosibirsk, 630090, Russia

E-mail address: dair@math.nsc.ru

Sobolev Institute of Mathematics, Universitetskil Pr., 4, Novosibirsk, 630090, Russia

E-mail address: sharaf@math.nsc.ru 